\title{
Differences in the Duration of Diuretic Effects and Impact on the Renin-Angiotensin- Aldosterone System of Furosemide in Healthy Dogs
}

\author{
Yasutomo HORI ${ }^{1) *}$, Naoko OHSHIMA ${ }^{1)}$, Kazutaka KANAI ${ }^{1)}$, Fumio HOSHI ${ }^{1)}$, Naoyuki ITOH $^{1)}$ and Sei-ichi HIGUCHI ${ }^{1)}$ \\ ${ }^{1)}$ Department of Small Animal Internal Medicine, School of Veterinary Medicine, Kitasato University, 23-35-1 Higashi, Towada, Aomori \\ 034-8628, Japan
}

(Received 10 June 2009/Accepted 18 August 2009/Published online in J-STAGE 5 November 2009)

\begin{abstract}
Our aim was to investigate the differences in the duration of diuretic effects and impact on the renin-angiotensin-aldosterone (RAA) system of furosemide as a model of short- and long-acting loop diuretics. Anesthetized dogs ( $\mathrm{n}=6$ ) were randomized into placebo, intravenous bolus administration (IB) and chronic rate infusion (CRI) groups. This study was conducted with a crossover study. Furosemide $(4 \mathrm{mg} / \mathrm{kg})$ was diluted to $18 \mathrm{~mL}$ in sterile saline. Furosemide was infused at $0.5 \mathrm{mg} / \mathrm{kg} / \mathrm{hr}$ for 8 hr in the CRI group or was injected at 0 and $4 \mathrm{hr}$ (both $2 \mathrm{mg} / \mathrm{kg}$ ) in the IB group. Blood and urine samples were collected at baseline and at 1, 2, 4, 5, 6 and $8 \mathrm{hr}$. Compared with the baseline, the IB group had a significantly increased urine output at 1 and $5 \mathrm{hr}$. The CRI group had a significantly increased urine output persisting for $4 \mathrm{hr}$ compared with the baseline. Compared with the placebo group, 8-hr urine output and 8-hr sodium excretion were significantly increased in the IB and CRI groups; the values in the CRI group were significantly higher than those in the IB group. Eight-hour potassium excretion was significantly increased in the IB and CRI groups. The plasma aldosterone concentration was significantly elevated in the IB group at $8 \mathrm{hr}$. Duration of action may be a predominant cause of loop diuretic-related differences. Persistent diuresis may cause greater diuretic effects than transient diuresis, with less elevation of the plasma aldosterone concentration.
\end{abstract}

KEY WORDS: canine, diuretic resistance, loop diuretics, plasma aldosterone concentrations.

J. Vet.Med.Sci. 72(1): 13-18, 2010

Loop diuretics are recommended as a first-line therapy in the management of congestive heart failure (CHF) and are important in the symptomatic treatment [19]. They can be divided into short-acting ones, such as furosemide, and long-acting ones, such as azosemide and torasemide. Although furosemide is commonly used in clinical settings, its main complication is that patients with CHF develop resistance to loop diuretics due to a reduction in the glomerular filtration rate and an increase in sodium reabsorption at diuretic-insensitive sites in the nephron $[6,10,11,28]$. Additionally, furosemide activates the renin-angiotensinaldosterone (RAA) system and the sympathetic nervous system $[14,16,32]$.

In contrast to furosemide, previous studies have revealed that long-acting loop diuretics have clinical efficacy; they cause less activation of the RAA system, reduce diuretic resistance, improve ventricular fibrosis and decrease the mortality rate in both humans and animal models [7, 17, 23, 37]. Furosemide treatment for 14 days leads to diuretic resistance in dogs, but this does not occur with torasemide [17]. In human clinical study, the myocardial collagen volume decreases with torasemide, but remains unchanged with furosemide [23]. Additionally, a recent study showed that torasemide is associated with lower mortality compared with furosemide in patients with CHF [7]. Similarly, our previous data suggested that azosemide causes mild and long-acting effects with minimal activation of the RAA sys-

\footnotetext{
* CoRrespondence to: Hori, Y., Department of Small Animal Internal Medicine, School of Veterinary Medicine, Kitasato University, 23-35-1 Higashi, Towada, Aomori 034-8628, Japan. e-mail: hori@vmas.kitasato-u.ac.jp
}

tem compared with furosemide in normal dogs [16]. In human patients with chronic heart failure, azosemide treatment significantly improves clinical symptoms and attenuates the plasma renin activity and norepinephrine concentrations as compared with furosemide treatment [32]. Compared with furosemide, azosemide improves ventricular fibrosis and decreases the mortality rate in a rat heart failure model [37]. Thus, long-acting loop diuretics are attractive in chronic management of heart failure.

However, pharmacological variability (dosage, absorption, half-life) can lead to differential diuretic effects and activity of the neurohumoral system [4, 5, 8, 13, 20, 29, 33]. In particular, torasemide is known to have anti-aldosteronergic effects [34], and azosemide is known to have anti-arginine vasopressin effects [26]. Thus, direct comparisons of the effects of different loop diuretics should be made with caution. Although a previous study reported that continuous infusion of furosemide induces greater diuresis than bolus administration in healthy dogs [1], direct comparisons of the RAA system related to different administration of furosemide remain unclear. In the present study, we administered the same dose of furosemide in the form of a bolus or a chronic rate infusion as models of short-acting and longacting loop diuretics, respectively. Our aim was to investigate differences in diuretic responses and the RAA system related to diuretic duration in anesthetized healthy dogs.

\section{MATERIALS AND METHODS}

Animals: Clinically healthy dogs $(\mathrm{n}=6)$ utilized as the subjects of this study. These dogs were adult mixed-breeds, 
aged 2-8 years, weighing $7-14 \mathrm{~kg}$; an equal number of dogs from both sexes were used ( 3 males and 3 females). These dogs were determined to be healthy on the basis of a complete physical examination and echocardiography. All dogs were housed individually in cages. The dogs were given commercial dry food and had free access to water. The Guidelines for Institutional Laboratory Animal Care and Use of the School of Veterinary Medicine at Kitasato University were followed during this study.

The dogs were sedated with butorphanol $(0.2 \mathrm{mg} / \mathrm{kg}$, iv $)$ and atropine $(0.025 \mathrm{mg} / \mathrm{kg}, \mathrm{sc})$, anesthetized with propofol $(6.0 \mathrm{mg} / \mathrm{kg}$, iv) and intubated. Anesthesia was maintained by a mixture of $2.0 \%$ isoflurane and oxygen. The end-tidal $\mathrm{PaCO}_{2}$ was monitored and maintained between 35 and 45 $\mathrm{mmHg}$, and the heart rate was monitored using an electrocardiogram (COLIN BP-608, Nihon Kohden, Tokyo, Japan). The dogs were positioned in left lateral recumbency. A 6-8 Fr Foley catheter was placed to collect urine samples. Following complete urination, blood and urine samples were collected after $2 \mathrm{hr}$, as a baseline.

In this crossover study, the dogs were randomized into placebo, intravenous bolus administration (IB) and chronic rate infusion (CRI) groups. In the placebo group, sterile saline $(18 \mathrm{ml} / \mathrm{dog})$ was administered for $8 \mathrm{hr}$ via the cephalic vein with an infusion pump. The same dose of furosemide (Lasix, Aventis Pharma, Tokyo, Japan; $4 \mathrm{mg} / \mathrm{kg}$ ) was diluted to $18 \mathrm{~m} l$ in sterile saline; it was administered in the form of IB or CRI. In the CRI group, furosemide was infused at $0.5 \mathrm{mg} / \mathrm{kg} / \mathrm{hr}$ for $8 \mathrm{hr}$ via the cephalic vein with an infusion pump. In the IB group, furosemide $(2 \mathrm{mg} / \mathrm{kg})$ was injected at 0 and $4 \mathrm{hr}$ via the cephalic vein. The total loading volume was equal in all groups. During drug treatment, blood and urine samples were collected at 1, 2, 4, 5, 6 and 8 $\mathrm{hr}$. The urine volume $(\mathrm{m} l / \mathrm{kg} / \mathrm{hr})$ and urine specific gravity (USG) were immediately measured. Each drug was given randomly, and the interval between different drugs was at least 7 days. After each washout period, each dog was then randomly assigned to receive one of the remaining treatments until each dog had received all three treatments.

Sample measurements: Two blood samples at each time point were drawn from the jugular vein, one with EDTA and one without an anticoagulant. The samples were then centrifuged $\left(3,000 \mathrm{rpm}, 4^{\circ} \mathrm{C}, 10 \mathrm{~min}\right)$, and the plasma and serum samples were stored at $-70^{\circ} \mathrm{C}$. Similarly, the collected urine samples were centrifuged $\left(1500 \mathrm{rpm}, 4^{\circ} \mathrm{C}, 5\right.$ $\mathrm{min})$, and the supernatant was stored at $-70^{\circ} \mathrm{C}$. The plasma protein concentration was measured by refractometry. The hematrocrit $(\mathrm{Ht} \%)$ was determined by the microhematocrit method. The blood urea nitrogen (BUN; $\mathrm{mg} / \mathrm{d} l$ ) and plasma creatinine $(\mathrm{mg} / \mathrm{d} l)$ concentrations were measured with an autoanalyzer (AU-400, Olympus Co, Tokyo, Japan). The plasma sodium, potassium and chloride concentrations $(\mathrm{mEq} / \mathrm{l})$ were measured with an electrolyte analyzer (EA06T, A\&T Co, Kanagawa, Japan). The plasma aldosterone concentrations were determined by radioimmunoassay (Aldosterone-RIA kit II, Yamasa Co, Chiba, Japan) [16]. Similarly, the plasma renin activities were determined by radioimmunoassay (SRL renin kit, TFB Co., Tokyo, Japan) [31]. The serum angiotensin converting enzyme (ACE) activities were determined by ELISA (ACE Color, Fujirebio Inc., Tokyo, Japan) [2]. Blood samples for measurement of the plasma aldosterone concentration, plasma renin activity and serum ACE activity were collected at $8 \mathrm{hr}$.

Statistical analyses: Data are described as means \pm SE. The time course changes in urine output and USG for each drug were compared with the baseline by one-factor repeated measures analysis of variance (ANOVA). The significance of the differences between the mean values of the baseline and each condition was tested with Tukey's multiple comparison test. One-way ANOVA was used to compare the results among the groups. The significance of the differences between the mean values of the placebo group and each condition was tested with Tukey's multiple comparison test. $P$ values $<0.05$ were considered to be statistically significant.

\section{RESULTS}

Compared with the baseline values, the heart rate was increased significantly by treatment with CRI at $4 \mathrm{hr}$ and by the placebo at $8 \mathrm{hr}$. Insignificant changes in the $\mathrm{Ht} \%$, plasma electrolytes, BUN and creatinine concentrations were observed among the groups at $8 \mathrm{hr}$ (Table 1). The CRI group had significantly increased plasma protein concentrations compared with the placebo group, but not in the IB group.

Compared with the baseline, the IB group had significantly increased urine output at 1 and $5 \mathrm{hr}$ [i.e., $1 \mathrm{hr}$ after administration of furosemide (both $P<0.001$; Fig. 1)]. The IB group had a significantly decreased USG compared with the baseline for $8 \mathrm{hr}$. In contrast, the CRI group had significantly increased urine output compared with the baseline $(P<0.001)$, and the increase persisted for $4 \mathrm{hr}$. The CRI group had a significantly decreased USG compared with the baseline for $8 \mathrm{hr}(P<0.001$, respectively).

The 8-hr urine output increased significantly in the IB and CRI groups compared with the placebo group (both $P<0.001$; Fig. 2). The 8-hr urine output of the CRI group was significantly higher than that of the IB group $(P<0.05)$. Similarly, the 8 -hr sodium excretion increased significantly

Table 1. $\mathrm{Ht} \%$ and plasma concentrations of several variables in 6 healthy dogs determined $8 \mathrm{hr}$ after administration of furosemide in a crossover study

\begin{tabular}{lccc}
\hline & Placebo & IB & CRI \\
\hline $\mathrm{Ht}(\%)$ & $40 \pm 1$ & $42 \pm 3$ & $41 \pm 2$ \\
$\mathrm{~T} . P .(\mathrm{g} / \mathrm{d} l)$ & $6.2 \pm 0.3$ & $6.7 \pm 0.1$ & $7.2 \pm 0.3^{*}$ \\
$\mathrm{BUN}(\mathrm{mg} / \mathrm{d} l)$ & $24.6 \pm 1.6$ & $29.9 \pm 1.9$ & $28.5 \pm 2.4$ \\
$\mathrm{Cre}(\mathrm{mg} / \mathrm{d} l)$ & $1.0 \pm 0.1$ & $1.1 \pm 0.1$ & $1.2 \pm 0.1$ \\
$\mathrm{Na}(\mathrm{mEq} / l)$ & $154 \pm 1$ & $154 \pm 1.1$ & $156 \pm 1.1$ \\
$\mathrm{~K}(\mathrm{mEq} / l)$ & $5.7 \pm 0.6$ & $5.0 \pm 0.4$ & $4.4 \pm 0.3$ \\
$\mathrm{Cl}(\mathrm{mEq} / l)$ & $118 \pm 1.2$ & $114 \pm 1.3$ & $111 \pm 1.5$ \\
\hline
\end{tabular}

Data are described as means $\pm \mathrm{SE}$.

T.P., plasma protein; BUN, blood urea nitrogen; Cre, creatinine. $* P<0.05$ vs. Placebo. 

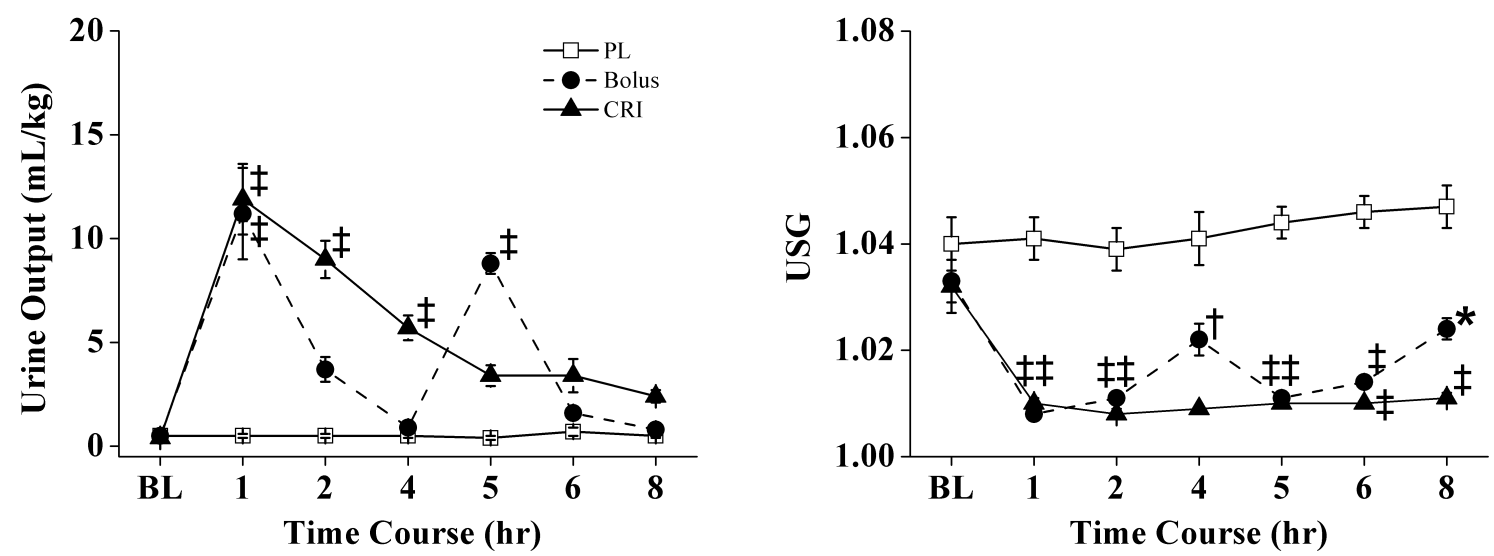

Fig. 1. Changes in urine volume (left panel) and specific gravity (right panel) in response to furosemide. Data are described as means \pm SE. * Value is significantly $(P<0.05)$ different from the baseline value. $\uparrow$ Value is significantly $(P<0.01)$ different from the baseline value. $\$$ Value is significantly $(P<0.001)$ different from the baseline value.

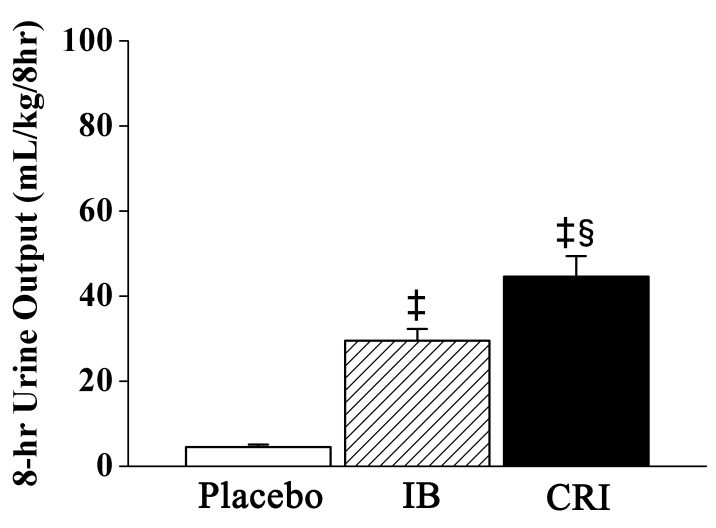

Fig. 2. The 8-hr urine volume determined after administration of placebo, intravenous bolus administration (IB) or chronic rate infusion of furosemide (CRI) in a crossover study. Data are described as means $\pm \mathrm{SE}$. $\$$ Value is significantly $(P<0.001)$ different from that for the placebo treatment. $\S$ Value is significantly $(P<0.05)$ different from that for the IB group.

in the IB and CRI groups compared with the placebo group (both $P<0.001$; Fig. 3 ). The 8 -hr sodium excretion in the CRI group was also significantly higher than that in the IB group $(P<0.05)$. Compared with the placebo group, 8-hr potassium excretion increased significantly in the IB and CRI groups $(P<0.05$ and 0.01 , respectively; Fig. 3$)$. After treatment for $8 \mathrm{hr}$, the plasma aldosterone concentration was significantly elevated in the IB group $(P<0.05)$ compared with the placebo group, but not in the CRI group (Fig. 4). The plasma renin activity and serum ACE activity were not significantly different among the groups.

\section{DISCUSSION}

Long-acting loop diuretics have beneficial effects in chronic management of heart disease [32, 37]. However, long-acting loop diuretics differ from furosemide in terms of pharmacological mechanism, oral bioavailability, absorption, affinity and biological half-life [4, 5, 8, 13, 20, 33]. Thus, direct comparisons of the effects of different loop diuretics should be made with caution. We investigated differences in diuretic responses and the RAA system related to diuretic duration in anesthetized healthy dogs.

The time course of furosemide delivery to the proximal renal tubule is an important determinant of diuretic effect $[15,36]$. Although an increasing plasma concentration of furosemide is related to increased renal clearance of the drug, once the furosemide concentration in the tubule exceeds a threshold, additional dosage increases will not further increase diuresis $[15,18]$. Lee et al. reported that when the same total dose of furosemide was administered as a bolus injection or continuous infusion in normal dogs, the plasma concentration of furosemide was immediately elevated and then decreased following a bolus injection, whereas the concentration was maintained at a lower level by continuous infusion [22]. In addition, they found that although pharmacokinetic parameters such as terminal halflife and renal clearance of furosemide were not significantly different among the groups in the study, urine output and sodium excretion increased in relation to the furosemide infusion times [22]. In human subjects, continuous infusion of furosemide results in greater diuresis than bolus therapy, despite the maximal plasma furosemide concentrations and amount of drug excreted in the urine being lower for continuous infusion than for bolus therapy $[9,36]$. In the present study, an increase in urine output was evident at $1 \mathrm{hr}$ after IB, but the response was transient. In contrast, urine output increased significantly after CRI, and the diuretic action persisted for $4 \mathrm{hr}$. Our study revealed that total urine output and natriuresis with CRI (i.e., persistent diuresis) were significantly greater than those with IB (i.e., temporary diuresis). These results are consistent with previous studies $[1,9$, 36]. Our results may provide basic information related to urine output and natriuresis as models of short- and longacting loop diuretics. 

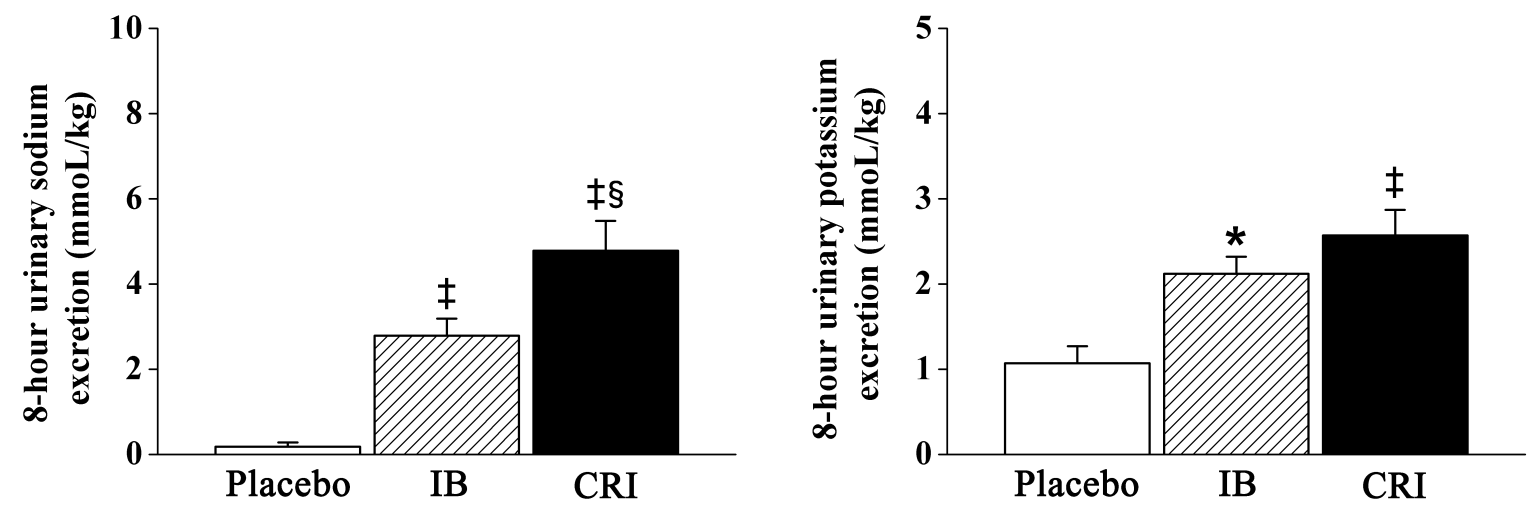

Fig. 3. The 8-hr urinary sodium excretion (left panel) and 8-hr urinary potassium excretion (right panel) determined after administration of placebo, intravenous bolus administration (IB) or chronic rate infusion of furosemide (CRI) in a crossover study. Data are described as means \pm SE. * Value is significantly $(P<0.05)$ different from that for the placebo treatment. $\ddagger$ Value is significantly $(P<0.001)$ different from that for the placebo treatment. $\S$ Value is significantly $(P<0.05)$ different from that for the IB group.
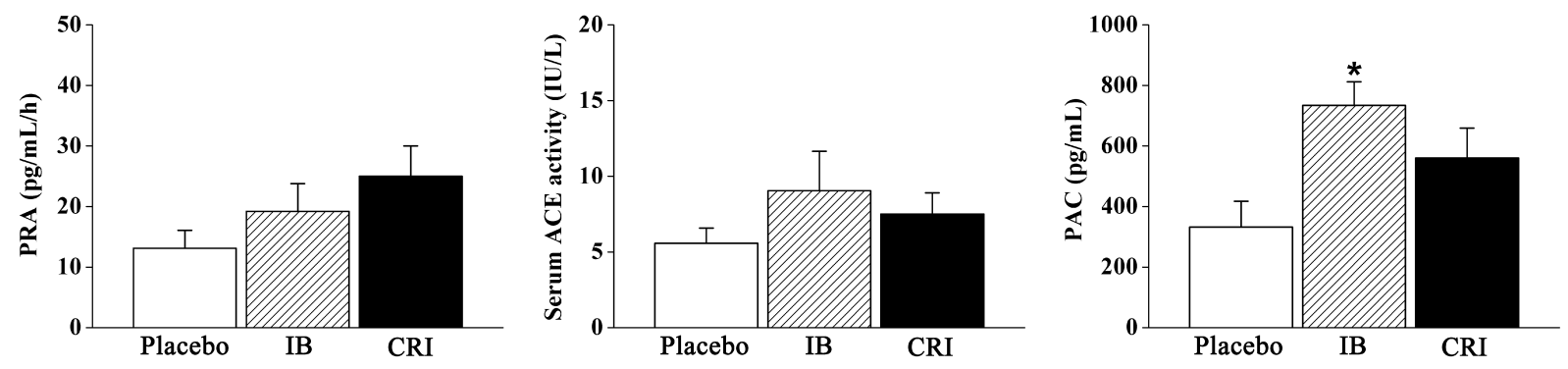

Fig. 4. The PAC, PRA and serum ACE activity values determined at $8 \mathrm{hr}$ after administration of placebo, intravenous bolus administration (IB) or chronic rate infusion of furosemide (CRI) in a crossover study. Data are described as means $\pm \mathrm{SE}$. * Value is significantly $(P<0.05)$ different from that for the placebo treatment.

Loop diuretic administration is associated with activation of neurohumoral axes such as the plasma concentrations of aldosterone, renin and norepinephrine [14, 24, 27, 32]. However, the response of the RAA system to furosemide differs with diuretic duration. In a previous report concerning patients with CHF who had been administered loop diuretics orally for more than 3 months, the plasma renin activity and norepinephrine concentrations were significantly higher with short-acting loop diuretics than with long-acting ones [32]. Although the plasma renin activity is immediately and temporally increased after intravenous administrations of furosemide in humans and dogs, it returns to baseline values over a few hours [14, 21, 25, 30]. In contrast, the plasma renin activity increases progressively to a maximum level $120 \mathrm{~min}$ after oral administrations of furosemide [25]. In the present study, although the total urine volume in the CRI group was significantly higher than in the IB group, the plasma aldosterone concentration was significantly higher in the IB group compared with the placebo group, but not in the CRI group. Both the plasma renin activity and serum ACE activity were slightly increased in the groups after $8 \mathrm{hr}$ of treatment, but the differences were not significant. These results indicated that persistent diuresis may cause greater diuretic effects than transient diuresis, with less elevation of the RAA system.

Furthermore, the time course response to furosemide differs for renin, angiotensin and aldosterone. Intravenous administration of furosemide markedly increased the plasma renin activity, norepinephrine concentration and angiotensin II concentration in the healthy dogs. After 120 min, the plasma renin activity decreased by half, but the plasma angiotensin II concentration remained high [30]. The plasma aldosterone concentration increased in response to furosemide; although this increase was similar following doses $>0.1 \mathrm{mg} / \mathrm{kg}$, the time to peak aldosterone response was dose dependent [27]. In addition, although the furosemide-induced increase in the plasma norepinephrine concentration and plasma renin activity may be mediated by the central sympathetic nervous system, the furosemideinduced increase in the plasma aldosterone concentration was not mediated [35]. In the present study, our results showed the different activation among the RAA system, i.e. the plasma aldosterone concentration, plasma renin activity and serum ACE activity, response to furosemide, which may be supported by previous reports.

The adverse effects of loop diuretics include natriuresis, kaliuresis and hypovolemia; these drugs may also induce dehydration, hypokalemia and azotemia $[3,12]$. In humans, 
chronic infusion of furosemide reduces the risk of adverse effects such as hypotension and ototoxicity due to lower peak serum concentrations [18]. In the present study, the CRI group showed significant increases in the total protein concentration and nonsignificant increases in the plasma BUN and potassium concentrations compared with the placebo and IB groups. Further studies are needed to investigate any adverse effects in dogs with heart disease.

Limitations: In the present study, our aim was not to investigate the time course response to loop diuretics in the RAA system, but to investigate differences of outcome in diuretic responses and the RAA system related to diuretic duration. Because different monitoring periods, measurement points and dosages may cause different results of RAA activation, our results should be interpreted with caution. We cannot exclude the possibility that general anesthesia may have caused hypotension that modulated the diuretic effects and activity of the RAA system. The coadministration of diuretics with an ACE inhibitor, digoxin, or a betaadrenergic antagonist might produce different effects. In addition, we did not examine the possibility that chronic heart disease may lead to different responses. Further study is warranted to address the long-term response to diuretics in dogs with heart disease.

Conclusions: The present study showed that CRI (persistent diuretics) of furosemide resulted in greater diuresis, despite a lower plasma aldosterone concentration than for IB (temporal diuretics). These results indicate that the duration of action is a predominant cause of loop diuretic-related differences, as well as pharmacological mechanisms. Additionally, our results may provide basic information about diuretic therapy, such as the different effects of short- and long-acting loop diuretics. Further studies to investigate the clinical usefulness of long-acting loop diuretics in dogs with heart disease are warranted.

\section{REFERENCES}

1. Adin, D.B., Taylor, A.W., Hill, R.C., Scott, K.C. and Martin, F.G. 2003. Intermittent bolus injection versus continuous infusion of furosemide in normal adult greyhound dogs. J. Vet. Intern. Med. 17: 632-636.

2. Aramaki, Y., Uechi, M. and Takase, K. 2003. Angiotensin converting enzyme and chymase activity in the feline heart and serum. J. Vet. Med. Sci. 65: 1115-1118.

3. Brater, D.C. 1996. Benefits and risks of torasemide in congestive heart failure and essential hypertension. Drug Saf. 14: 104-120.

4. Brater, D.C. 1979. Renal sites of action of azosemide. Clin. Pharmacol. Ther. 25: 428-434.

5. Brater, D.C., Day, B., Anderson, S. and Seiwell, R. 1983. Azosemide kinetics and dynamics. Clin. Pharmacol. Ther. 34: 454-458.

6. Cataliotti, A., Boerrigter, G., Costello-Boerrigter, L.C., Schirger, J.A., Tsuruda, T., Heublein, D.M., Chen, H.H., Malatino, L.S. and Burnett, J.C., Jr. 2004. Brain natriuretic peptide enhances renal actions of furosemide and suppresses furosemide-induced aldosterone activation in experimental heart failure. Circulation 109: 1680-1685.
7. Cosin, J. and Diez, J. 2002. Torasemide in chronic heart failure: results of the TORIC study. Eur. J. Heart Fail. 4: 507513.

8. Delarge, J. 1988. Chemistry and pharmacological properties of the pyridine-3-sulfonylurea derivative torasemide. Arzneimittelforschung 38: 144-150.

9. Dormans, T.P., van Meyel, J.J., Gerlag, P.G., Tan, Y., Russel, F.G. and Smits, P. 1996. Diuretic efficacy of high dose furosemide in severe heart failure: bolus injection versus continuous infusion. J. Am. Coll. Cardiol. 28: 376-382.

10. Ellison, D.H. 1999. Diuretic resistance: physiology and therapeutics. Semin. Nephrol. 19: 581-597.

11. Ellison, D.H. 2001. Diuretic therapy and resistance in congestive heart failure. Cardiology 96: 132-143.

12. Flamenbaum, W. and Friedman, R. 1982. Pharmacology, therapeutic efficacy, and adverse effects of bumetanide, a new "loop" diuretic. Pharmacotherapy 2: 213-222.

13. Fowler, S.F. and Murray, K.M. 1995. Torsemide: a new loop diuretic. Am. J. Health Syst. Pharm. 52: 1771-1780.

14. Francis, G.S., Siegel, R.M., Goldsmith, S.R., Olivari, M.T., Levine, T.B. and Cohn, J.N. 1985. Acute vasoconstrictor response to intravenous furosemide in patients with chronic congestive heart failure. Activation of the neurohumoral axis. Ann. Intern. Med. 103: 1-6.

15. Hammarlund, M. and Paalzow, L.K. 1985. Acute tolerance development to the diuretic effect of furosemide in the rat. Biopharm. Drug Dispos. 6: 9-21.

16. Hori, Y., Katou, A., Tsubaki, M., Kanai, K., Nakao, R., Hoshi, F., Itoh, N. and Higuchi, S. 2008.Assessment of diuretic effects and changes in plasma aldosterone concentration following oral administration of a single dose of furosemide or azosemide in healthy dogs. Am. J. Vet. Res. 69: 1664-1669.

17. Hori, Y., Takusagawa, F., Ikadai, H., Uechi, M., Hoshi, F. and Higuchi, S. 2007. Effects of oral administration of furosemide and torsemide in healthy dogs. Am. J. Vet. Res. 68: 1058-1063.

18. Howard, P.A. and Dunn, M.I. 2006. Effectiveness of continuous infusions of loop diuretics for severe heart failure. J. Cardiovasc. Med. (Hagerstown) 7: 5-10.

19. Hunt, S.A., Abraham, W.T., Chin, M.H., Feldman, A.M., Francis, G.S., Ganiats, T.G., Jessup, M., Konstam, M.A., Mancini, D.M., Michl, K., Oates, J.A., Rahko, P.S., Silver, M.A., Stevenson, L.W., Yancy, C.W., Antman, E.M., Smith, S.C., Jr., Adams, C.D., Anderson, J.L., Faxon, D.P., Fuster, V., Halperin, J.L., Hiratzka, L.F., Jacobs, A.K., Nishimura, R., Ornato, J.P., Page, R.L. and Riegel, B. 2005. ACC/AHA 2005 Guideline update for the diagnosis and management of chronic heart failure in the adult: A report of the american college of cardiology/american heart association task force on practice guidelines (Writing committee to update the 2001 guidelines for the evaluation and management of heart failure): developed in collaboration with the American college of chest physicians and the international society for heart and lung transplantation: endorsed by the heart rhythm society. Circulation 112: e154235.

20. Kuzuya, F. 1983.Phase 1 study of azosemide (SK-110): singleand multiple-dose study. Int. J. Clin. Pharmacol. Ther. Toxicol. 21: 10-23.

21. Lammintausta, R., Anttila, M. and Viinamaki, O. 1980. Response of renin, aldosterone and antidiuretic hormone to furosemide and furosemide-triamterene combination. Int. J. Clin. Pharmacol. Ther. Toxicol. 18: 395-398.

22. Lee, M.G., Li, T. and Chiou, W.L. 1986. Effect of intravenous infusion time on the pharmacokinetics and pharmacodynamics 
of the same total dose of furosemide. Biopharm. Drug Dispos. 7: 537-547.

23. Lopez, B., Querejeta, R., Gonzalez, A., Sanchez, E., Larman, M. and Diez, J. 2004. Effects of loop diuretics on myocardial fibrosis and collagen type I turnover in chronic heart failure. $J$. Am. Coll. Cardiol. 43: 2028-2035.

24. McCurley, J.M., Hanlon, S.U., Wei, S.K., Wedam, E.F., Michalski, M. and Haigney, M.C. 2004. Furosemide and the progression of left ventricular dysfunction in experimental heart failure. J. Am. Coll. Cardiol. 44: 1301-1307.

25. Noda, Y., Fukiyama, K., Kumamoto, K., Takishita, S., Eto, T., Kawasaki, T. and Omae, T. 1982. Renin response to furosemide differs with the routes of administration in health men. Jpn. Circ. J. 46: 552-558.

26. Osajima, A., Anai, H., Segawa, K., Muta, T., Takasugi, M. and Kuroiwa, A. 1992. Comparative effects of loop diuretics on AVP-receptor binding and AVP-sensitive adenylate cyclase activity. Nippon Jinzo Gakkai Shi 34: 965-972.

27. Patel, A. and Smith, F.G. 1997. Dose-dependent cardiovascular, renal, and endocrine effects of furosemide in conscious lambs. Can. J. Physiol. Pharmacol. 75: 1101-1107.

28. Paterna, S., Di Pasquale, P., Parrinello, G., Amato, P., Cardinale, A., Follone, G., Giubilato, A. and Licata, G. 2000. Effects of high-dose furosemide and small-volume hypertonic saline solution infusion in comparison with a high dose of furosemide as a bolus, in refractory congestive heart failure. Eur. J. Heart Fail. 2: 305-313.

29. Suh, O.K., Kim, S.H. and Lee, M.G. 2003. Pharmacokinetics and pharmacodynamics of azosemide. Biopharm. Drug Dispos. 24: 275-297.

30. Suzuki, H., Saruta, T. and Ferrario, C.M. 1986. Changes in central and peripheral renin-angiotensin system after furo- semide injection. Endocrinol, Jpn. 33: 497-503.

31. Takai, S., Sakonjo, H., Fukuda, K., Jin, D., Sakaguchi, M., Kamoshita, K., Ishida, K., Sukenaga, Y. and Miyazaki, M. 2003. A novel chymase inhibitor, 2-(5-formylamino-6-oxo-2phenyl-1,6-dihydropyrimidine-1-yl)-N-[[,4-dioxo-1-phenyl-7(2-pyridyloxy)]2-heptyl]acetamide (NK3201), suppressed intimal hyperplasia after balloon injury. J. Pharmacol. Exp. Ther. 304: 841-844.

32. Tsutsui, T., Tsutamoto, T., Maeda, K. and Kinoshita, M. 2001. Comparison of neurohumoral effects of short-acting and longacting loop diuretics in patients with chronic congestive heart failure. J. Cardiovasc. Pharmacol. 38 (Suppl.1): S81-85.

33. Uchida, T., Ohtaki, Y., Kido, H. and Watanabe, M. 1991. Diuretic profile of a novel loop diuretic torasemide in rats and dogs. Drugs Exp. Clin. Res. 17: 293-298.

34. Uchida, T., Yamanaga, K., Nishikawa, M., Ohtaki, Y., Kido, H. and Watanabe, M. 1991. Anti-aldosteronergic effect of torasemide. Eur. J. Pharmacol. 205: 145-150.

35. Ueno, Y., Arita, M., Suruda, H., Mohara, O. and Masuyama, Y. 1983. Contributions of central sympathetic neural activity to furosemide-induced increases in plasma renin activity and noradrenaline. Jpn. Heart J. 24: 259-267.

36. van Meyel, J.J., Smits, P., Russel, F.G., Gerlag, P.G., Tan, Y. and Gribnau, F.W. 1992. Diuretic efficiency of furosemide during continuous administration versus bolus injection in healthy volunteers. Clin. Pharmacol. Ther. 51: 440-444.

37. Yoshida, J., Yamamoto, K., Mano, T., Sakata, Y., Nishio, M., Ohtani, T, Hori, M., Miwa, T. and Masuyama, T. 2005. Different effects of long- and short-acting loop diuretics on survival rate in Dahl high-salt heart failure model rats. Cardiovasc. Res. 68: $118-127$. 